\title{
Patología quirúrgica de la glándula tiroides
}

\author{
Surgical pathology of the thyroid gland
}

\author{
Juan Oré ${ }^{1}$, José Saavedra ${ }^{2}$ \\ ${ }^{1}$ Médico Ex Residente. Servicio Cirugía de Cabeza y Cuello. Hospital Dos De Mayo. Lima. Perú. \\ ${ }^{2}$ Jefe del Servicio de Cirugía Cabeza y Cuello. Hospital Dos De Mayo. Lima. Perú.
}

\begin{abstract}
Resumen
Introducción: Debido a la gran diversidad de patología quirúrgica de la glándula tiroides y su alta frecuencia en nuestro medio, creemos conveniente presentar nuestra experiencia en esta patología. Objetivos: Conocer la incidencia de la patología quirúrgica de la glándula tiroides, según diagnóstico anatomopatológico, en pacientes operados. Diseño: Estudio retrospectivo, observacional y descriptivo. Lugar: Servicio de cirugía de cabeza y cuello, Hospital Dos de Mayo, Lima, Perú, hospital de enseñanza médica. Participantes: Pacientes operados de la glándula tiroides. Intervenciones: Se revisó las historias clínicas de pacientes operados de la glándula tiroides, desde enero de 1997 hasta diciembre de 2006. Principales medidas de resultados: Resultados anatomopatológicos en los especímenes de glándula tiroides. Resultados: Hubo 274 casos operados, de los cuales $81,4 \%$ del total correspondió al sexo femenino; $56,9 \%$ de los casos provenía de fuera de Lima y Callao, con edades que fluctuaban desde los 30 y 59 años. El tumor fue el signo predominante en $97,8 \%$ de los casos. La operación realizada más frecuente fue la tiroidectomía total $(39,8 \%)$. La patología benigna representó $58 \%$ de los casos, con el adenoma folicular como principal patología benigna ( $23 \%$ ); dentro de las neoplasias malignas ( $42 \%$ del total), $23,7 \%$ era carcinoma papilar. Conclusiones: La patología tumoral tiroidea presentó un marcado predominio en el sexo femenino, principalmente en los grupos etáreos de la $4^{\mathrm{a}}, 5^{\mathrm{a}}$ y $6^{\mathrm{a}}$ décadas. La patología glandular tiroidea fue en su mayoría benigna, y de la maligna, el carcinoma papilar. Con alto valor de especificidad $(97,7 \%)$ y valor predictivo positivo $(95,4 \%)$, la biopsia de aspiración por aguja fina de tiroides continúa siendo el examen auxiliar más importante en el estudio del paciente con patología tumoral tiroidea.

Palabras clave: Patología quirúrgica; glándula tiroides; cirugía; neoplasias.
\end{abstract}

\begin{abstract}
Introduction: Due to the great diversity and variety of thyroid surgical pathology in our country, we review our experience with this pathology. Objectives: To determine the incidence of thyroid gland surgical pathology in patients undergoing surgery. Design: Descriptive and retrospective study. Setting: Head and neck service, Hospital Dos de Mayo, Lima, Peru, a teaching hospital. Participants: Patients undergoing surgery of the thyroid gland. Interventions: Clinical histories and surgical reports involving the thyroid gland in the period January 1997 through December 2006 were reviewed. Main outcome measures: Pathology results of thyroid gland surgical specimens. Results: Two hundred and seventy four cases had thyroid surgery, $81,4 \%$ females; $56,9 \%$ of the cases corresponded to Lima, capital of Peru. Incidence was higher between 30 and 59 years of age. Growing tumor was the most frequent sign with $97,8 \%$. Total thyroidectomy was the most frequent surgery $(39,8 \%)$. Benign pathologies represented $58 \%$ of the cases, with follicular adenoma as the main pathology $(23 \%)$; and for malignant tumors ( $42 \%$ from the total) papillary carcinoma was the main neoplasia $(23,7 \%)$. Conclusions: Surgical pathology of the thyroid gland had higher incidence in women, mainly in the $4^{\text {th }}, 5^{\text {th }}$ and $6^{\text {th }}$ age decades. Thyroid tumors were by far benign and the main malignant tumor was papillary carcinoma. Fine needle aspiration biopsy continues to be the most important auxiliary study in the patient with a thyroid tumor, with high specificity value $(97,7)$ and positive predictive value $(95,4)$.
\end{abstract}

Key words: Pathology, surgical; thyroid gland; surgery; neoplasms.

\section{INTRODUCCIÓN}

La patología tumoral tiroidea tiene variedad de presentaciones en nuestro medio, habiéndose descrito crecimiento de la glándula ya sea en forma difusa o nodular y pudiendo localizarse en una glándula hiperfuncionante, hipofuncionante o en una glándula con función hormonal normal. Existen diferentes modalidades de tratamiento y pronóstico, así como carcinoma tiroideo, adenomas, quistes, tiroiditis y metástasis en zonas no endémicas.

La forma más frecuente de presentación es el nódulo tiroideo, el cual se presenta en cerca de $4 \%$ en personas entre 30 y 50 años, $1,5 \%$ en niños y adolescentes y $5 \%$ en personas alrededor de los 60 años. Tiene una frecuencia de presentación en el sexo femenino de $94 \%$ y de $6 \%$ en el masculino; aunque, es en varones donde la mayor cantidad de los nódulos son malignos ${ }^{(1-4)}$. Se caracteriza por un nódulo único asintomático, comúnmente encontrado en el examen físico. Aunque pueden llegar a ser hiperfuncionante, catalogándose así como bocio nodular tóxico, aun así, la mayoría de los nódulos tiroideos corresponde a adenomas simples (80\%). En el bocio nodular tóxico o enfermedad de Plummer se encuentra un nódulo hipercaptador responsable de la clínica de hipertiroidismo ${ }^{(4)-7)}$. Figuras 1 y 2.

Los quistes tiroideos representan 15 a $25 \%$ de todos los tumores tiroideos y se evidencian en la gammagrafía como un nódulo hipocaptador, en una glándula normofuncionante. La gran mayoría de los quistes es macronodular, que sufren degeneración con acumulación de fluido seroso, sangre o una sustancia coloide ${ }^{(7,8)}$. Aproximadamente, cerca de 5\% de la población mundial presenta bocio, con una frecuencia de hasta 6 veces más en las mujeres. El bocio endémico es definido como el agrandamiento generalizado de la tiroides, por deficiencia dietética de iodo, a diferencia del bocio esporádico, el cual se presenta en áreas sin déficit de iodo. Se considera bocio multinodular a una etapa tardía de un bocio difuso, debido a la atrofia o necrosis de áreas glandulares, con hipertrofia del tejido remanente, como compensación. Ello difiere del Graves-Basedow, de diferente etiología, donde existe hipertiroidismo con bocio difuso, oftalmopatía y dermopatía (mixedema) ${ }^{(2-4,6,8)}$. El bocio puede llegar a presentar una extensión endotorácica, compresión traqueal, disfagia, síndrome de compresión de la vena cava superior, parálisis del nervio recurrente o síndrome de Horner ${ }^{(4,8,9)}$.

Las lesiones inflamatorias son denominadas tiroiditis, las cuales se caracterizan 


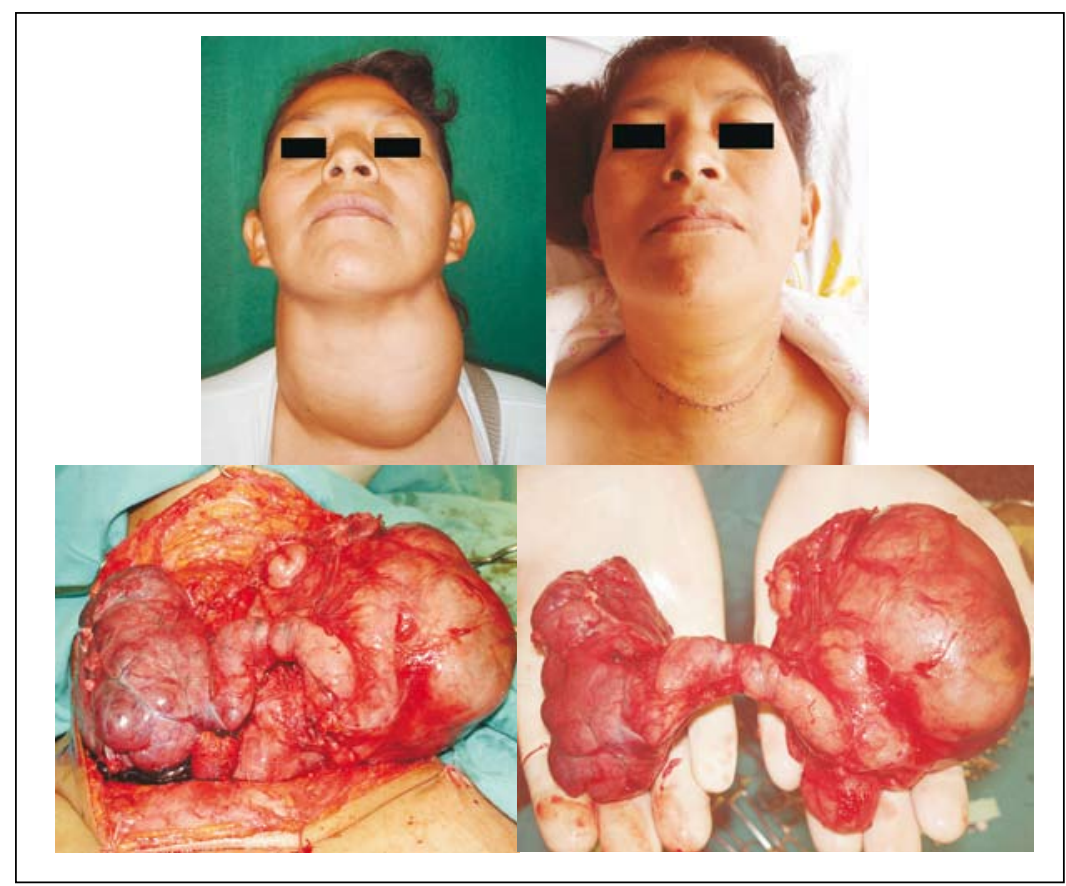

Figura 1. Paciente con patología benigna tiroidea. Mujer de 45 años con adenoma folicular según anatomía patológica. Vista del campo quirúrgico y de la pieza operatoria en manos del cirujano.

por ser dolorosas en su forma aguda (infecciosa) e indolora en su forma crónica. La tiroiditis crónica linfocítica o de Hashimoto es la más frecuente. Tiene un origen autoinmune, debido a autoanticuerpos bloqueantes de la TSH, antiperoxidasa y antitiroglobulina, con edad de presentación entre 30 y 50 años en mujeres y de 40 a 50 años en varones, con mayor predominio -en un $90 \%$ - en las mujeres ${ }^{(2,4,6,10)}$. Existe una secuencia de hipertiroidismo y finalmente hipotiroidismo, a diferencia de la tiroiditis subaguda de D'Quervain, la cual suele presentarse luego de una infección viral de las vías respiratorias altas; en esta, la clínica es trifásica, con hipertirodismo, hipotiroidismo y la última fase euti- roidea, según los niveles hormonales circulantes. En la tiroiditis subaguda, la gammagrafía muestra una captación baja y los anticuerpos antitiroideos son habitualmente negativos. La tiroiditis de Riedel o tiroiditis fibrosa es la forma menos frecuente, con una consistencia pétrea e hipotiroidismo, causando disfagia, disnea ${ }^{(4,7,11)}$.

La malignidad para los nódulos solitarios y los bocios multinodulares es 5 a $12 \%$ y $3 \%$, respectivamente. Dentro de los cánceres de tiroides, tenemos carcinomas diferenciados (90 a 95\% ) e indiferenciados $(5 \text { a } 10 \%)^{(2,4.5)}$. El carcinoma papilar es el más frecuente ( 76 a 80\%) de todos los carcinomas tiroideos diferen- ciados; es el principal tipo de carcinoma tiroideo en niños ( $75 \%$ ) y en expuestos a radiación (80 a 90\%). Usualmente, es un nódulo solitario indoloro, aunque puede involucrar a toda la glándula, en 30\%. La edad promedio de presentación es de 30 a 40 años, con una relación de 3 a 1 en mujeres frente a varones. Es el menos agresivo del resto de los carcinomas, con buen pronóstico. Su metástasis se debe a una diseminación vía linfática a ganglios cervicales, siendo rara las metástasis a distancia vía hematógena, (10\%) y de localización en pulmones y huesos ${ }^{(2-5,8)}$. Figuras 3 y 4.

El carcinoma folicular es el segundo en frecuencia; es más agresivo que el carcinoma papilar, con igual edad de presentación, predominio en mujeres y clínica, que el anterior. Las metástasis ganglionares son menores al 10\%. La propagación es más hematógena que linfática y va a huesos y pulmones. Tiene una variante, el carcinoma de células Hürtle, que tiene receptores para TSH y produce tiroglobulina ${ }^{(2-4,13)}$.

El tratamiento en pacientes con carcinoma diferenciado metastásico (10 a 15\% al momento del diagnóstico) es la tiroidectomía, con disección ganglionar (en caso de compromiso ganglionar cervical), seguida de iodoablación (por compromiso extraglandular) y hormonosupresión (para suprimir el eje hipófisis-tiroides y, posteriormente, como terapia de mantenimiento). Los valores de recurrencia están entre 15 a $25 \%$ de los pacientes; sin embargo, el pronóstico es alentador, $90 \%$ a los 20 años ${ }^{(4,5,8)}$.

El carcinoma medular tiroideo (4 a $5 \%)$ surge de las células parafoliculares

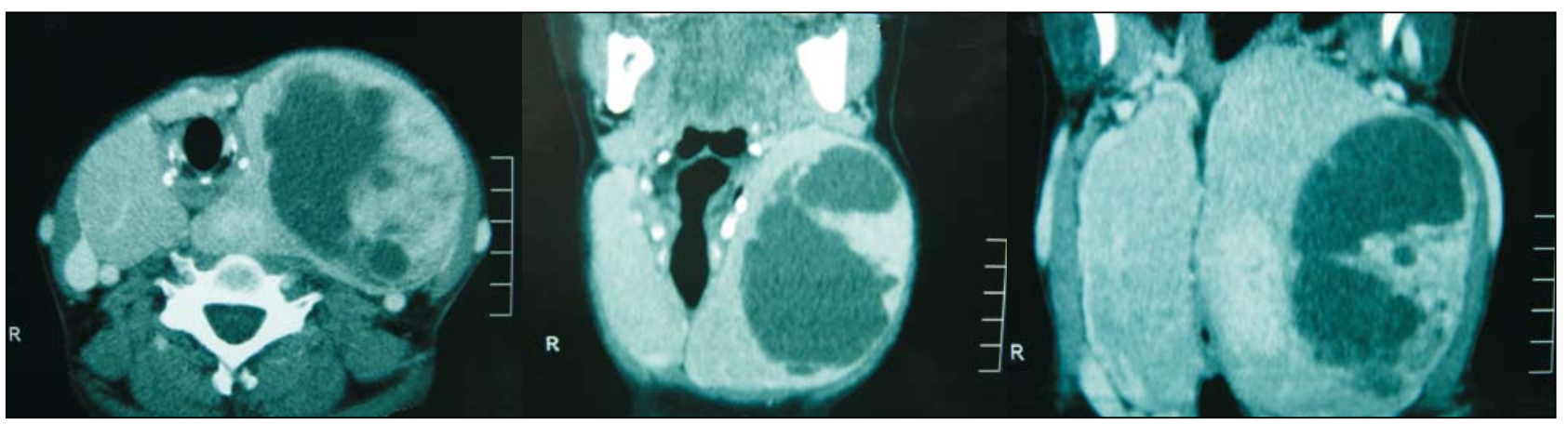

Figura 2. Tomografía espiral multicorte cervical del paciente del caso anterior. Nótese el desplazamiento de la vía respiratoria ocasionada por el lóbulo tiroideo izquierdo. Lóbulo derecho también aumentado de tamaño. 


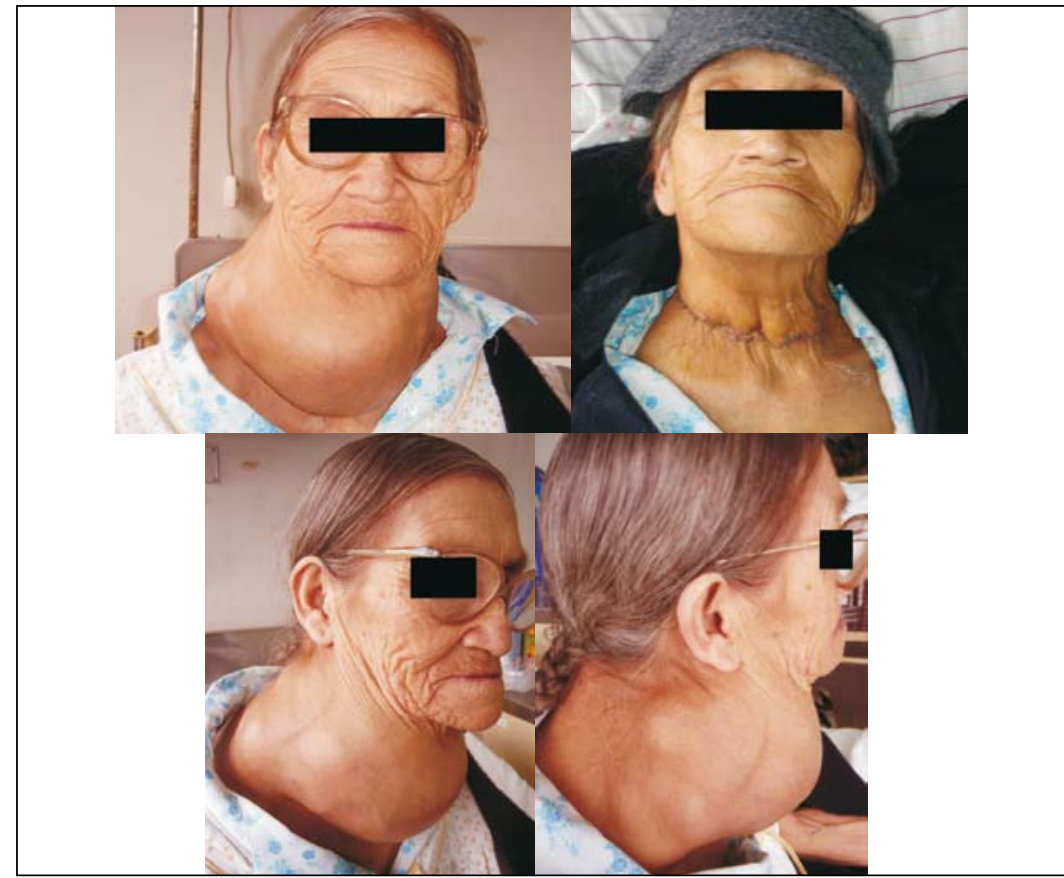

Figura 3. Paciente con patología maligna tiroidea. Mujer de 80 años con carcinoma papilar según anatomía patológica. Acude por tumor, disfagia, disfonía con disnea ocasional.

de la tiroides, que producen calcitonina (su marcador tumoral), y tiene como localización embriológica la parte media a superior de los lóbulos tiroideos, sin presentar diferencia significativa según sexos; cerca de 25 a $33 \%$ de los casos son hereditarios y el resto, esporádico. En lo hereditario, pueden encontrarse asociados a la neoplasia endocrina múltiple tipo 2, siendo la NEM 2A la más frecuente (carcinoma medular tiroideo, feocromocitoma e hiperparatiroidismo), con relación a la NEM 2B (carcinoma medular de tiroides, feocromocitoma, habito marfanoide y neuromas mucosos). El hereditario no asociado a NEM es denominado carcinoma medular familiar. La edad típica de presentación en la forma esporádica es entre 50 y 60 años y en la forma asociada a la NEM-2, entre 20 y 30 años. Es unilateral en $75 \%$ y la mayoría doloroso, con disfonía, disfagia o disnea. Pueden presentarse síndromes paraneoplásicos, como síndrome carcinoide, con palpitaciones, flushing y diarrea, en $30 \%$. Esta neoplasia, al no ser producto de la célula tiroidea que metaboliza el iodo, no es sensible al iodo radioactivo ${ }^{(1-6)}$.

El carcinoma anaplásico o indiferenciado $(1 \%)$ se origina de un tumor diferenciado de larga data, sin preferencia por sexos y con mayor incidencia entre los 60 y 80 años. Es el más agresivo de todos los carcinomas; es frecuente la asociación con disfonía, disfagia y/o disnea y la presentación de metástasis regionales y a distancia. No concentra iodo, de importancia para el tratamiento postoperatorio ${ }^{(2,3,5,12)}$.

El linfoma tiroideo en su mayoría es no Hodgkin de células B; buena cantidad de linfomas se asocian luego o durante una tiroiditis de Hashimoto. Es más frecuente en mujeres, principalmente entre los 55 y 75 años. La sobrevida a 5 años es de $89 \%$, si la enfermedad es diagnosticada en forma precoz, y $5 \%$ si tiene la forma diseminada ${ }^{(1,6-8)}$. Las metástasis son infrecuentes, manifestación tardía de un cáncer no primario de la glándula; son más frecuentes el hipernefroma y el carcinoma broncogénico ${ }^{(2,4)}$.

Creemos necesario presentar nuestra experiencia de las diversas patologías tiroideas en el Hospital Nacional Dos de Mayo y ponerla en conocimiento de los médicos del Perú ${ }^{(1-3)}$.

\section{MÉTODOS}

Se realizó un estudio descriptivo retrospectivo en el que se revisó todas las historias clínicas y los reportes operatorios de los pacientes sometidos a cirugía electiva de la glándula tiroides. La población se obtuvo del registro de cirugías del servicio de Cirugía de Cabeza y Cuello, en el periodo comprendido entre enero del 1997 y diciembre 2006, del Hospital Nacional Dos de Mayo, Lima. Perú.

Se incluyó todos los pacientes intervenidos de la glándula tiroides, cuya pieza quirúrgica fue dicha glándula en su totalidad o en forma parcial. Además, se

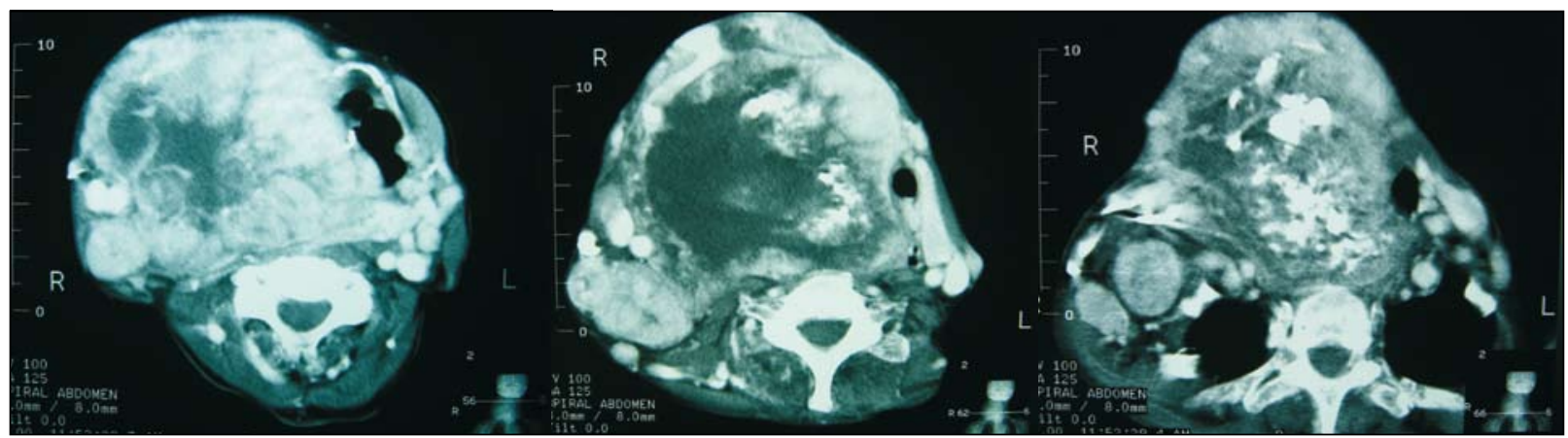

Figura 4. Tomografía espiral multicorte cervical del caso anterior. Se aprecia el gran tamaño de la glándula tiroides y el desplazamiento de las estructuras vecinas tanto grandes vasos y vía aerodigestiva. 
revisó el resultado anatomopatológico. Los criterios de exclusión fueron aquellos pacientes con historias clínicas con datos incompletos, perdidos o inconsistentes. Se consideró las siguientes variables: edad, sexo, síntomas y signos al ingreso, diagnóstico preoperatorio, operación realizada, complicaciones y resultado del informe anatomopatológico.

Se identificó las historias clínicas a revisar y se procedió a llenar los datos en documento de recolección de datos, elaborándose una hoja de recolección de datos para los resultados obtenidos, siendo procesados con el programa estadístico de Microsoft Excel 2007.

\section{RESULTADOS}

Se revisó las historias clínicas de 274 pacientes intervenidos quirúrgicamente, en forma electiva o ambulatoria, de patología tiroidea, entre los periodos enero 1997 y diciembre 2006. Del total, 223 pacientes fueron del sexo femenino $(81,4 \%)$ y 51 del sexo masculino (18,6\%).

La mayor cantidad de pacientes acudió de Lima y Callao, con 43,1\%, seguido de los departamentos de Junín, 9,5\%, Huancavelica, 9,1\%, Huancayo, 8\%, Ayacucho, 6,9\%, y Cajamarca, 6\%. Ver Tabla 1.

La edad varió entre 12 y 83 años para los varones y entre 15 y 90 años para las mujeres, con una media de 50,7 y 46,7 años para varones y mujeres, respectivamente. La mayor incidencia se presentó entre los 50 y 59 años $(20,4 \%)$, teniendo a continuación a los grupos de 30 a 39

\begin{tabular}{|c|c|c|c|c|c|c|}
\hline \multirow[b]{2}{*}{ Procedencia } & \multicolumn{2}{|c|}{ Masculino } & \multicolumn{2}{|c|}{ Femenino } & \multicolumn{2}{|c|}{ Total } \\
\hline & $\mathrm{n}$ & $\%$ & $\mathrm{n}$ & $\%$ & $\mathrm{n}$ & $\%$ \\
\hline Áncash & 1 & 2,0 & 2 & 0,9 & 3 & 1,1 \\
\hline Arequipa & 1 & 2,0 & 3 & 1,3 & 4 & 1,5 \\
\hline Apurimac & 3 & 5,9 & 8 & 3,6 & 11 & 4,0 \\
\hline Ayacucho & 3 & 5,9 & 16 & 7,2 & 19 & 6,9 \\
\hline Cajamarca & 4 & 7,8 & 14 & 6,3 & 18 & 6,6 \\
\hline Cerro de Pasco & 3 & 5,9 & 6 & 2,7 & 9 & 3,3 \\
\hline Cusco & 0 & 0,0 & 9 & 4,0 & 9 & 3,3 \\
\hline Huancavelica & 7 & 13,7 & 18 & 8,1 & 25 & 9,1 \\
\hline Huancayo & 6 & 11,8 & 16 & 7,2 & 22 & 8,0 \\
\hline Ica & 0 & 0,0 & 0 & 0,0 & 0 & 0,0 \\
\hline Junín & 5 & 9,8 & 21 & 9,4 & 26 & 9,5 \\
\hline Lima / Callao & 15 & 29,4 & 103 & 462 & 118 & 43,1 \\
\hline Puno & 3 & 5,9 & 7 & 3,1 & 10 & 3,6 \\
\hline Total & 51 & 100,0 & 223 & 100,0 & 274 & 100,0 \\
\hline
\end{tabular}

años $(19,7 \%)$ y 40 a 49 años (19,3\%). Hubo distribución similar en el sexo femenino, con $60,1 \%$ de los casos entre los 30 y 59 años de edad; para el sexo masculino, $60,8 \%$ de los casos tuvo entre 40 y 69 años. Ver Tabla 2.

Tabla 2. Grupos etáreos según sexo.

\begin{tabular}{lrrrrrr}
\hline & \multicolumn{2}{c}{ Masculino } & \multicolumn{2}{c}{ Femenino } & \multicolumn{2}{c}{ Total } \\
Edad & $\mathrm{n}$ & \multicolumn{1}{c}{$\%$} & \multicolumn{1}{c}{$\mathrm{n}$} & \multicolumn{1}{c}{$\%$} & \multicolumn{1}{c}{$\mathrm{n}$} & $\%$ \\
\hline$<20$ años & 1 & 2,0 & 6 & 2,7 & 7 & 2,6 \\
$20-29$ años & 6 & 11,8 & 31 & 13,9 & 37 & 13,5 \\
30 - 39 años & 6 & 11,8 & 48 & 21,5 & 54 & 19,7 \\
$40-49$ años & 11 & 21,6 & 42 & 18,8 & 53 & 19,3 \\
$50-59$ años & 12 & 23,5 & 44 & 19,7 & 56 & 20,4 \\
$60-69$ años & 8 & 15,7 & 31 & 13,9 & 39 & 14,2 \\
$70-79$ años & 6 & 11,8 & 15 & 6,7 & 21 & 7,7 \\
$>79$ años & 1 & 2,0 & 6 & 2,7 & 7 & 2,6 \\
Total & 51 & 100,0 & 223 & 100,0 & 274 & 100,0 \\
\hline
\end{tabular}

Del cuadro clínico asociado, el signo principal por el cual acudieron los pacientes fue el aumento de volumen de la glándula tiroides, en $97,8 \%$ de los casos, seguido de la disfagia y disfonía, en $8,8 \%$ y $6,9 \%$, respectivamente.

De total de los casos intervenidos, el diagnóstico preoperatorio más frecuente fue bocio nodular, $38 \%$, seguido del carcinoma tiroideo y el bocio multinodular con $10,2 \%$ en cada uno e igual distribución en el sexo femenino. Para el sexo masculino, el bocio nodular representó $37,3 \%$ y el carcinoma tiroideo, $25,5 \%$ de los casos. Ver Tabla 3.

En los exámenes auxiliares, por medio de la ecografía, en 49,6\% se describía un único nódulo sólido en la glándula tiroides, en 22,3\% bocio multinodular, en $20,4 \%$ bocio difuso y en $7,7 \%$ quiste tiroideo. Solo $6,9 \%$ tenía un perfil tiroideo

compatible con hipertiroidismo y $2,2 \%$ con hipotiroidismo; así, 90,9\% era eutiroideo al momento de la cirugía.

De los resultados de biopsia aspiración guiada con aguja fina (BAAF), 30 casos fueron catalogados como no específi$\cos (10,9 \%), 28$ casos $(10,2 \%)$ como neoplasia folicular; $61 \mathrm{ca}$ sos fueron catalogados como positivos para neoplasia maligna, $22,3 \%$, de los cuales $4(1,5 \%)$ fueron descritos como carcinoma medular en la BAAF. Se obtuvo 122 casos negativos $(44,5 \%)$ y se describió quiste tiroideo en 29 casos $(10,6 \%)$. Ver Figura 5.

El procedimiento quirúrgico más realizado fue la tiroidectomía total, en $39,8 \%$, la hemitiroidectomía, en 31,4\%, y la tiroidectomía subtotal, en $20,1 \%$. Se realizó disección ganglionar cervical en $6,6 \%$ de los casos. Dicho orden de frecuencia fue similar para ambos sexos. Ver Figura 6.

Obtuvimos 161 casos benignos (58\%) frente a 113 casos de malignos (42\%). De la patología benigna tiroidea, 84,5\% (161 casos) correspondió al sexo femenino y 15,5\% (25 casos) al sexo masculino. En la distribución de la patología maligna, también el predominio fue del sexo femenino, con $77 \%$ (113 casos), frente a 23\% (26 casos) para varones. Y en la distribución de neoplasias benignas versus malignas para cada sexo, el $61 \%$ de los casos femeninos (136 casos) correspondió a patología benigna tiroidea, siendo el $39 \%$ (87 casos) neoplasias malignas. En el caso del sexo masculino, obtuvimos $49 \%$ ( 25 casos) y $51 \%$ (26 casos), respectivamente.

De los resultados anatomopatológicos, en general, el diagnóstico benigno más frecuente fue el adenoma folicular, tanto en el sexo masculino, femenino y en total, con $27,5 \%, 22 \%$ y $23 \%$, respectivamente; seguido del bocio multinodular, $13,1 \%$, y el bocio coloide, 11,6\%. De las patología malignas, el carcinoma papilar de tiroides sin metástasis fue el de mayor incidencia, en 23,7\% del total de cáncer tiroideo. En segundo lugar, el carcinoma medular de

Tabla 3. Diagnóstico preoperatorio según casos.

\begin{tabular}{lrrrrrr}
\hline & \multicolumn{2}{c}{ Masculino } & \multicolumn{2}{c}{ Femenino } & \multicolumn{2}{c}{ Total } \\
& $\mathrm{n}$ & \multicolumn{1}{c}{$\%$} & $\mathrm{n}$ & $\%$ & $\mathrm{n}$ & $\%$ \\
\hline Bocio difuso & 7 & 13,7 & 19 & 8,5 & 19 & 6,9 \\
Bocio nodular & 19 & 37,3 & 104 & 46,6 & 104 & 38,0 \\
Bocio multinodular & 2 & 3,9 & 28 & 12,6 & 28 & 10,2 \\
Bocio endotorácico & 2 & 3,9 & 7 & 3,1 & 7 & 2,6 \\
Quiste de tiroides & 0 & 0,0 & 4 & 1,8 & 4 & 1,5 \\
Carcinoma de tiroides & 13 & 25,5 & 28 & 12,6 & 28 & 10,2 \\
Carcinoma medular & 0 & 0,0 & 5 & 2,2 & 5 & 1,8 \\
Carcinoma de tiroides & & & & & & \\
con metástasis & 5 & 9,8 & 15 & 6,7 & 15 & 5,5 \\
Metástasis ganglionar & 1 & 2,0 & 0 & 0,0 & 0 & 0,0 \\
Tiroiditis & 0 & 0,0 & 1 & 0,4 & 1 & 0,4 \\
Plummer & 1 & 2,0 & 3 & 1,3 & 3 & 1,1 \\
Graves Basedow & 1 & 2,0 & 9 & 4,0 & 9 & 3,3 \\
Total & 51 & 100,0 & 223 & 100,0 & 274 & 100,0 \\
\hline
\end{tabular}




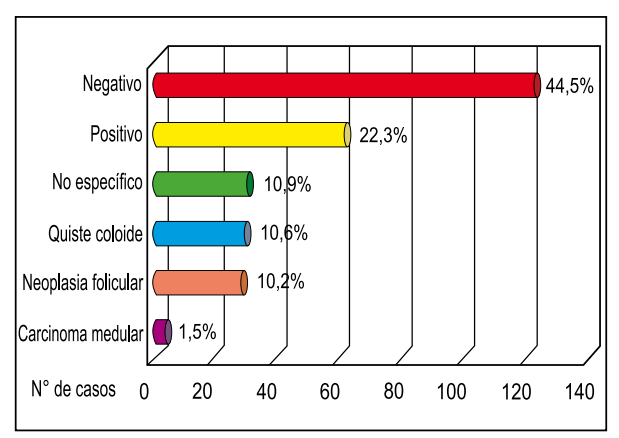

Figura 5. Resultados de la biopsia aspiración guiada por aguja fina.

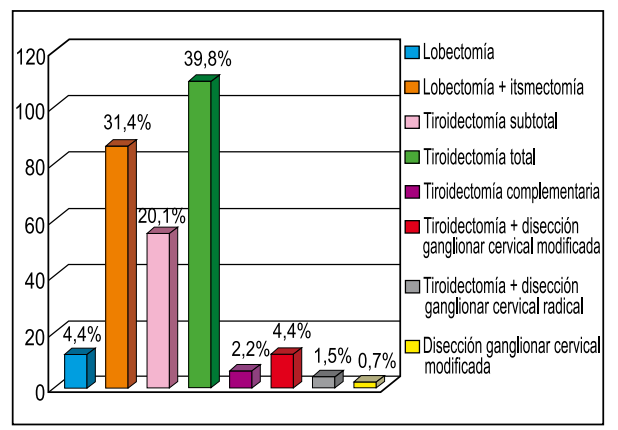

Figura 6. Operaciones realizadas.

tiroides, 12 casos $(4,4 \%)$, y el folicular, 5 casos $(1,8 \%)$. Ver Tabla 4.

Obtuvimos 74 pacientes con complicaciones postoperatorias, de 274 casos operados, es decir, $27 \%$ del total de pacientes, entre las cuales la disfonía fue la mayor, en 34 casos $(12,4 \%)$; cabe resaltar que todas fueron en carcinoma papilar. Fue seguida de la hipocalcemia transitoria, 7,3\% (20 casos), y la hipocalcemia permanente, con 5,8\% (16 casos). Solo $2(0,7 \%)$ casos fueron reintervenidos para control de hemostasia, por sangrado postoperatorio (ambos carcinoma medular). La fístula cervical y la infección del sitio operatorio se presentó en carcinoma papilar. Ver Figura 7.

\section{DISCUSIÓN}

Los resultados del presente estudio guardan relación con los que se describe en la literatura, tanto en el predominio de la patología glandular en el sexo femenino como a la edad de presentación. El aumento de volumen de la glándula tiroides fue el signo de mayor frecuencia, de los cuales $49 \%$ se presentó como bocio nodular unilateral. De los bocios difusos $(20,4 \%)$ y multinodulares $(22,3 \%)$, el $16 \%$ y $41 \%$, respectivamente, fueron neoplasias malignas ${ }^{(2-4)}$. El 90,9\% de los pacientes era eutiroideo, tanto en carcinomas como neoplasias benignas, no haciendo mayor diferencia en el estudio preoperatorio, salvo en los casos de hiperfunción; el estudio fue solicitado al servicio de endocrinología.

En cuanto a la biopsia aspiración con aguja fina (BAAF), encontramos valores de sensibilidad de $72,1 \%$ y de especificidad $97,7 \%$, con un valor predictivo positivo y negativo de $95,4 \%$ y $84,1 \%$, respectivamente. ${ }^{(3-5,5,12,13)}$

Tabla 4. Resultado anatomopatológico según sexo.

\begin{tabular}{lrrrrrr}
\hline & \multicolumn{2}{c}{ Masculino } & \multicolumn{2}{c}{ Femenino } & \multicolumn{2}{c}{ Total } \\
& $\mathrm{n}$ & \multicolumn{1}{c}{$\%$} & $\mathrm{n}$ & \multicolumn{1}{c}{$\%$} & $\mathrm{n}$ & $\%$ \\
\hline Adenoma folicular & 14 & 27,5 & 49 & 22,0 & 63 & 23,0 \\
Adenoma quístico & 3 & 5,9 & 12 & 5,4 & 15 & 5,5 \\
Bocio multinodular & 2 & 3,9 & 34 & 15,2 & 36 & 13,1 \\
Bocio coloide simple & 1 & 2,0 & 21 & 9,4 & 22 & 8,0 \\
Bocio coloide degenerado & 2 & 3,9 & 8 & 3,6 & 10 & 3,6 \\
Enfermedad de Graves Basedow & 1 & 2,0 & 2 & 0,9 & 3 & 1,1 \\
Carcinoma a células claras & 0 & 0,0 & 1 & 0,4 & 1 & 0,4 \\
Carcinoma papilar de tiroides & 13 & 25,5 & 52 & 23,3 & 65 & 23,7 \\
Carcinoma papilar, variante folicular & 6 & 11,8 & 6 & 2,7 & 12 & 4,4 \\
Carcinoma papilar metastásico de tiroides & 1 & 2,0 & 10 & 4,5 & 11 & 4,0 \\
Carcinoma folicular de tiroides & 2 & 3,9 & 3 & 1,3 & 5 & 1,8 \\
Carcinoma medular de tiroides & 1 & 2,0 & 11 & 4,9 & 12 & 4,4 \\
Carcinoma medular metastásico de tiroides & 0 & 0,0 & 2 & 0,9 & 2 & 0,7 \\
Carcinoma anaplásico & 2 & 3,9 & 2 & 0,9 & 4 & 1,5 \\
Linfoma a células medianas a grandes & 1 & 2,0 & 0 & 0,0 & 1 & 0,4 \\
Tiroiditis crónica fibrosada inespecífica & 0 & 0,0 & 3 & 1,3 & 3 & 1,1 \\
Tiroiditis crónica granulomatosa & 1 & 2,0 & 0 & 0,0 & 1 & 0,4 \\
Tiroiditis linfocítica de Hashimoto & 1 & 2,0 & 7 & 3,1 & 8 & 2,9 \\
Total & 51 & 100,0 & 223 & 100,0 & 274 & 100,0 \\
\hline & & & & & & \\
\hline
\end{tabular}

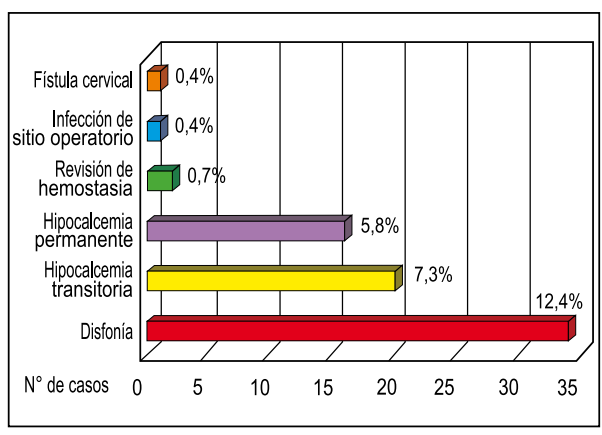

Figura 7. Complicaciones postoperatorias.

Se describe ligero predominio en la patología benigna tiroidea frente a la maligna ( $58 \%$ frente a $42 \%$ ), siendo la tiroidectomía total destinada a los casos de cáncer tiroideo y en ciertos casos de benignidad, con la hemitiroidectomía y la tiroidectomía subtotal para el resto de patologías. Todas tuvieron disección ganglionar, obviamente, en casos con compromiso metastásico regional, con terapia adicional de iodoablación, radioterapia coadyuvante y hormonosupresión $(2,4,10,11)$.

Los adenomas tiroideos constituyeron la patología benigna más frecuentemente encontrada, tanto en su distribución en general, como para cada sexo. De las tiroiditis, la de Hashimoto fue la de mayor presentación, con mayor incidencia en el sexo femenino.

Del cáncer tiroideo, con iguales resultados que otros estudios, el carcinoma papilar fue el predominante de los carcinomas y del total de neoplasias malignas tiroideas, seguida del carcinoma folicular y medular, con resultados según sexo y edades similar a los de la literatura. Hubo contados casos de carcinoma anaplásico y linfoma tiroideo ${ }^{(3,4,11)}$.

En cuanto a las complicaciones, se presentaron en mayor cantidad en pacientes con neoplasias malignas. Debido a que la mayor cantidad de casos fueron carcinomas papilares, la mayor cantidad de complicaciones se presentó en estos casos, siendo las complicaciones de mayor gravedad en pacientes medulares y anaplásicos ${ }^{(8,12,13)}$.

Como conclusión, la patología quirúrgica tiroidea, tuvo predominio en el sexo femenino, al igual que en los grupos etáreos correspondientes a la $4^{\mathrm{a}}, 5^{\mathrm{a}}$ y $6^{\mathrm{a}}$ décadas, 
variables de suma importancia a tener en cuenta, al igual que lo descrito en la literatura.

La patología glandular tiroidea fue en su mayoría benigna, pero no podemos dejar de lado a la patología maligna, teniendo como principal tipo el carcinoma papilar. El carcinoma papilar tiroideo presenta, como se describe en la literatura, mejor pronóstico entre los carcinomas diferenciados, además de ser el tipo más frecuente dentro de los cánceres de tiroides.

La biopsia por aspiración con aguja fina de los tumores tiroideos es el examen auxiliar más importante en el estudio de un paciente, debido a su alto valor de especificidad y valor predictivo positivo.

\section{AGRADECIMIENTOS}

Al servicio de Cirugía de Cabeza y Cuello del Hospital Dos de Mayo, por otorgar el apoyo necesario durante la investigación.

\section{REFERENCIAS BIBLIOGRÁFICAS}

1. Niederhuber JE, Chen H. Surgical management of endocrine malignancies. Surgical Oncology Clinics of North America. Philadelphia: Editorial Saunders; 2006.

2. Harrison LB, Sessions RB, Ki Hong W. Head and neck cancer. $2^{\text {nd }}$ edition. Philadelphia: Editorial Lippincott Williams \& Wilkins; 2004.

3. Shah JP, Patel SG. Cirugía y oncología de cabeza y cuello. $3^{a}$ edición. Madrid: Editorial Mosby; 2004.

4. Myers EN, Suen JY, Myers JN. Cancer of the head and neck. $4^{\text {th }}$ edition. Philadelphia: Editorial Saunders; 2003.

5. Clark OH, Quan-Yang D, Siperstein AE. Cirugía endocrina. Clínicas Quirúrgicas de Norteamérica. Nueva York: Editorial McGraw-Hill; 1995.

6. Greenfield L, Mulholland MW, Oldhan KT. Head and neck. En: Surgery: Scientific principles and practice. $3^{\text {rd }}$ edition. Philadelphia: Editorial Lippincott Williams \& Wilkins; 2001.

7. Novelli JL, Piazza MV, Sánchez A. Patología quirúrgica de la glándula tiroides. $1^{\text {ra }}$ edición. Rosario, Argentina: Editorial UNR; 1997.

8. Avello A. Cirugía de cabeza, del cuello y maxilofacial. $1^{\text {era }}$ edición. Lima: Editorial MAD; 2002.
9. Ariyan S. Cancer of the head and neck. $1^{\text {st }}$ edition. St. Louis: Mosby Editorial; 1987.

10. Romero Torres R. Tratado de cirugía. $3^{\text {ra }}$ edición. Lima: Editorial Medicina Moderna; 2000.

11.Swarthz S. Principios de cirugía. $7^{\text {ma }}$ edición. México: Editorial McGraw-Hill; 2000.

12. Way LW, Doherty GM. Current surgical diagnosis and treatment. $11^{\text {th }}$ edition. New York: McGraw-Hill; 2003.

13. Dworkin HJ, Meier DA, Kaplan M. Advances in the management of patients with thyroid disease. Semin Nucl Med. 1995;25(3):205-20.

14. Avello A. Cáncer de tiroides. En: Barboza E. Principios y Terapéutica Quirúrgica. $1^{\text {ra }}$ edición. Lima: Universidad Peruana Cayetano Heredia; 1999. p. 496.

Manuscrito recibido el 10 de julio de 2008 y aceptado para publicación el 15 de agosto de 2008.

Correspondencia:

Dr. Juan Francisco Oré Acevedo

Av. Paseo la Castellana 620, Dpto. 102.

Lima 33, Perú

Correo-e: juanfcoore@yahoo.com 